\title{
CORRIGENDUM
}

\section{Singular robust room-temperature spin response from topological Dirac fermions}

Lukas Zhao, Haiming Deng, Inna Korzhovska, Zhiyi Chen, Marcin Konczykowski, Andrzej Hruban, Vadim Oganesyan and Lia Krusin-Elbaum

Nature Materials 13, 580-585 (2014); published online 18 May 2014; corrected after print 22 May 2014.

In the version of this Letter originally published, in Fig. 3a, the values of $n$ should have read ' $\sim 10^{19} \mathrm{~cm}^{-3}$ ' and ' $\sim 10^{17} \mathrm{~cm}^{-3}$. This error has now been corrected in the online versions of the Letter. 